\title{
Increasing the Number of Synapses Modifies Olfactory Perception in Drosophila
}

\author{
Angel Acebes and Alberto Ferrús \\ Instituto Cajal, Consejo Superior de Investigaciones Científicas, Madrid E-28002, Spain
}

The Drosophila mutant gigas produces an enlargement of postmitotic cells caused by additional rounds of DNA replication. In neurons, the mutant cell establishes more synapses than normal. We have taken advantage of this feature to study the effect of synapse number on odorant perception. Mosaic adults were generated in which one antenna was homozygous for gigas, whereas the contralateral side served as an internal control. Morphological analysis indicates that the number and type of sensory afferents forming the mutant antenna, as well as their projection to the olfactory glomeruli, are normal. In contrast, the volume of identified glomeruli increases to a variable extent, and mutant sensory neurons branch profusely. The number of synapses, estimated in the ventral (V) glomerulus that receives ipsilateral afferents only, is increased twofold to threefold. Large-dense-core vesicle-containing terminals that probably

Nervous systems detect and process environmental features, generating and storing complex information with great accuracy. These properties result from the regulation of cell and synapse number, both during development and in activity-dependent processes (Goodman and Shatz, 1993; Vaux and Korsmeyer, 1999). Since the early postulate of Hebb (1949) that memory formation is based on changes in the number of effective synapses, the experimental evidences have been somewhat controversial about the morphological changes while more supportive about the functional modulation (Moser, 1999). Nonetheless, neural branching and synaptogenesis are clearly related to usage in many species (Valverde, 1967; Innocenti et al., 1977; Bailey and Kandel, 1993; Heisenberg et al., 1995; Rajan and Cline, 1998; Maguire et al., 2000). It is reasonable to assume that the large repertoire of behaviors exhibited by large brains is sustained by a correspondingly large number of synapses. The supporting experimental evidence, however, is technically difficult to obtain. Thus far, attempts to modify the number of synapses, $N$, to study potential effects on behavior have been based on functional inactivation (Sweeney et al., 1995) or surgical removal strategies, that is, decreasing $N$. Here, we studied sensory perception effects after increasing $N$ after a genetic procedure.

To that end, we used the mutant gigas (gig) of Drosophila

\footnotetext{
Received Jan. 10, 2001; revised April 9, 2001; accepted April 19, 2001.

This research was funded by Grants PM99-099 from the Ministry of Technology and 8.5/43/1998 from the Comunidad Autónoma de Madrid (Spain). We appreciate the critical comments from lab members and our colleagues of the Cajal Institute. The assistance of Dr. R. Rodríguez and C. Bailón at the EM and confocal facilities of the Cajal Institute is also acknowledged. Dr. M. Martínez-Padron provided key training for EAG recordings.

Correspondence should be addressed to A. Ferrús, Instituto Cajal, Consejo Superior de Investigaciones Científicas, Avenida Doctor Arce 37, 28002 Madrid, Spain. E-mail: aferrus@cajal.csic.es.

Copyright (C) 2001 Society for Neuroscience $\quad 0270-6474 / 01 / 216264-10 \$ 15.00 / 0$
}

modulate olfactory centers are identified in the $\mathrm{V}$ glomerulus. Their number and size are not modified by the mutant input. Sensory transduction, measured by electroantennograms, is normal in amplitude and kinetics. In odorant tests, however, the profile of the behavioral response to ethyl acetate shows attractive responses to concentrations to which sibling controls remain indifferent $\left(10^{-8}\right.$ and $\left.10^{-7} \mathrm{v} / \mathrm{v}\right)$. In addition, the intensity of the response is augmented both at attractive and repulsive odorant concentrations with respect to that of controls. These results demonstrate that increased synapse number in the sensory neurons can modify the behavior of the organism, allowing a higher sensitivity of perception.

Key words: olfaction; sensory perception; gigas; synapse number; olfactory glomeruli; Drosophila
(Ferrús and García-Bellido, 1976). The relevant trait of this lethal mutant is that homozygous cells are substantially larger than normal because of additional rounds of DNA replication after completion of the normal mitotic program (Canal et al., 1998). The gene encodes a homolog of the human TSC2, a protein involved in a benign form of tumor that contains giant cells (Ito and Rubin, 1999). In mosaic eyes, mutant photoreceptors establish up to three times more synapses on genetically normal interneurons of the first optic neuropil, the lamina, than do adjacent normal photoreceptors (Canal et al., 1994). The phototactic response of these mosaics differs from normal. Wild-type flies follow a straight path toward the light source, whereas gig mosaics describe a helical path, exposing the normal eye to the light source (Canal et al., 1994). This qualitative observation suggested that gig photoreceptors convey an excessive or modified light input. Unfortunately, the distortion of corneal lenses produced by the mutation makes the visual system unsuitable for quantitative behavioral assays, and the olfactory system seems better suited for this purpose. The structural organization of this sensory system is quite similar in vertebrates and invertebrates (Hildebrand and Shepherd, 1997). In Drosophila, most olfactory sensillae are located in the antennal funiculus, and each includes one to four sensory neurons (Stocker, 1994; Shanbhag et al., 1995). These neurons express unique odorant receptor types, of the 57 known so far (Vosshall et al., 2000), and project via the antennal nerve (AN) to the primary olfactory neuropil, the antennal lobe (AL). This structure is divided into 43 glomeruli (Laissue et al., 1999) that are functional subunits involved in olfactory processing. Sensory projections converge into specific glomeruli according to the odorant receptor expressed, generating an odotopic map (Gao et al., 2000). Stimulus processing yields spatial representations based on the combinatorial activation of glomeruli in insects 
(Rodrigues and Pinto, 1989; Joerges et al., 1997; Galizia et al., 1999) as well as in mammals (Friedrich and Korsching, 1998; Rubin and Katz, 1999). Finally, these maps are thought to translate into odorant-specific patterns of coincident synaptic activity (Laurent, 1999).

\section{MATERIALS AND METHODS}

Fly strains and genetic procedures. The gigas gene is represented by three independently induced alleles, gig $^{109}$, gig $^{25}$, and gig $^{8 C 5}$; the first two were induced by ethyl-methane-sulfonate, and the last appeared spontaneously. All alleles show virtually the same phenotype. Genetic mosaics were obtained by mitotic recombination after x-ray irradiation of the following genotypes: (1) mwh jv gig ${ }^{109} \mathrm{red}$ ro/M(3) ${ }^{i 55}$; Gal4-72OK/UNG6, (2) $m w h$ jv gig 109 red ro/M(3) ${ }^{i 55}$, and (3) Gal4-C155; UNG6/+; mwh jv gig $^{109} \mathrm{red} \mathrm{rol}^{\mathrm{M(3)} i 55}$. Irradiation was delivered between 0 and $48 \mathrm{hr}$ of development at a dosage of $500 \mathrm{rad}$ (Philips MG 102; $150 \mathrm{rad} / \mathrm{min}, 100$ $\mathrm{kV}, 15 \mathrm{~mA}, 2 \mathrm{~mm} \mathrm{Al} \mathrm{filter).} \mathrm{Only} \mathrm{mosaics} \mathrm{with} \mathrm{a} \mathrm{whole} M^{+}$gig antenna were selected for this study. The extent of the mutant clone was determined by the $m w h$ and $j v$ markers that become homozygous in the same recombination event as gig. Behavioral experiments and quantitative EM observations were performed on 3- to 5-d-old flies. Antennae from wild-type Canton-S flies $(C-S)$ were used as controls for sensillae and axonal countings. In electrophysiological and behavioral experiments, irradiated $m w h$ jv gig ${ }^{109}$ red $r o / M(3)^{i 55}$ siblings without visible clones were used as genetic controls.

Light microscopy procedures. To count sensillae, whole antennae from control $(C-S)(n=6)$ and mosaic $(n=3)$ flies were mounted in a glycerol-PBS $(1: 1 \mathrm{v} / \mathrm{v})$ solution, between two coverslips allowing access to both sides of the antenna. The number and distribution of sensillae were obtained from drawings using a camera lucida coupled to a Nikon Optiphot light microscope. For histology, mosaic $(n=11)$ and control $(n=15)$ heads were fixed overnight at $4^{\circ} \mathrm{C}$ in alcoholic Bouin's $(10 \%$ formaldehyde, $6.7 \%$ glacial acetic acid, $0.44 \% \mathrm{w} / \mathrm{v}$ picric acid, and $50 \%$ ethanol) and embedded in paraffin. Serial sections of $10 \mu \mathrm{m}$, obtained on a Reichert-Jung 2050 microtome, were silver-impregnated by the Holmes-Blest method (Blest and Davie, 1980). Golgi impregnations were as described (Bausenwein et al., 1992). Photographs were taken with a Zeiss Axiophot light microscope.

Visualization and volumetric measurements of glomeruli. We used the Gal4/UAS system (Perrimon, 1998; Phelps and Brand, 1998) to visualize glomeruli in the antennal lobe. The UAS line UNG6 expresses the green fluorescent protein (GFP) coupled to the synaptic protein $n$-synaptobrevin, and $\mathrm{C} 155$ is a pan-neural Gal4 line (Stimson and Ramaswami, 1999); both lines were kindly provided by Dr. M. Ramaswami (Tucson University, Tucson, AZ). Line Gal4-72OK labels sensory afferents projecting to a subset of glomeruli in the antennal lobe (VM1, VM4, and DL1) (referred to as Gal4 line C in Ferveur et al., 1997), and was kindly provided by Dr. C. O'Kane (Cambridge University, Cambridge, UK). Dissected brains from mosaic $(n=8)$ and nonmosaic $(n=6)$ individuals of genotypes Gal4-72OK/UNG6; mwh jv gig $^{109}$ red ro/M(3) $)^{i 55}$ and Gal4-C155; UNG6/+; mwh jv gig ${ }^{109}$ red rol $M(3)^{i 55}$ were mounted in PBS and observed as whole mounts in a confocal microscope (Leica, Nussloch, Germany; TLS4D) using a fluorescein filter block, with the krypton-argon laser light source set for excitation at $488 \mathrm{~nm}$. Serial optical sections of $1 \mu \mathrm{m}(512 \times 512$ pixels $)$ were obtained to generate volumetric measurements using the Q500 software (Leica). The CorelDraw computer program was used to draw glomerular contours, and Q500 was used to measure areas from these contours.

Electron microscopy. To analyze antennal nerves, heads $(n=5)$ were fixed overnight at $4^{\circ} \mathrm{C}$ (4\% paraformaldehyde, $1 \%$ glutaraldehyde in 0.1 $\mathrm{M}$ phosphate buffer, $\mathrm{pH}$ 7.2) after removal of the proboscis. After several washes in $0.1 \mathrm{M}$ phosphate buffer, heads were post-fixed in $2 \% \mathrm{OsO}_{4}$ in phosphate buffer for $45 \mathrm{~min}$ at $4^{\circ} \mathrm{C}$ in the dark, dehydrated in a graded ethanol series, and included in Araldite resin. Ultrathin silver sections $(60-70 \mathrm{~nm})$ from a Reichert Ultracut E ultramicrotome were collected on Formvar-coated, single-slot grids and stained with uranyl acetate (10 min) and lead citrate $(10 \mathrm{~min})$. Micrographs were obtained in a JEOL 1200 EX electron microscope, and large paper copies of scanned negatives were used for counting axons. To analyze the number of synapses in the antennal neuropil, the fixative was modified (1\% paraformaldehyde, $2.5 \%$ glutaraldehyde in $0.1 \mathrm{M}$ phosphate buffer, $\mathrm{pH} 7.2$ ), as were the counterstaining solutions and incubation times. Here we used uranyl acetate (2\% in double distilled water, $1 \mathrm{hr}$ ) and lead citrate (15 min). These modifications yield a better visualization of synaptic specializations. Using semithin frontal sections, the ventral (V) glomerulus was localized in each antennal lobe $(n=8)$, and ultrathin serial sections were taken at this level. This glomerulus receives sensory afferents from the ipsilateral antenna only and, thus, the comparison between contralateral homologs provides an internal control for the mutant effect. Synapse quantification was performed by the disector technique (Sterio, 1984; West, 1999). Alternate ultrathin sections were established as the suitable distance between disectors because, by our estimations, a synaptic specialization never spans beyond two adjacent sections $(140 \mathrm{~nm})$. In each mosaic, electron micrographs obtained from both $\mathrm{V}$ glomeruli were printed at the same magnification, coded, and randomized for blind analysis. A total of 25-30 disectors were analyzed for each V glomerulus in each mosaic. The same disectors were used to estimate the number of boutons containing large-dense-core vesicles in this glomerulus.

Electroantennograms. Electroantennogram (EAG) recordings were obtained as previously described (Alcorta, 1991; Ayer and Carlson, 1992) in mosaics $(n=5)$ and sibling controls $(n=6)$. Extracellular responses from gig and contralateral third antennal segments were simultaneously recorded using two low-resistance $(\approx 5 \mathrm{M} \Omega)$ unbroken pulled glass electrodes $(1 \mu \mathrm{m} \varnothing)$. Electrodes were apposed on the cuticle and filled with a Drosophila Ringer's solution (in mM: $100 \mathrm{NaCl}, 5 \mathrm{KCl}, 20 \mathrm{MgCl}_{2}, 0.15$ $\mathrm{CaCl}_{2}, 5$ HEPES, 115 sucrose, and 5 trehalose). A reference electrode was inserted into the hemolymph, near the proboscis. Odorant stimulation was delivered by an electronic pump device in a continuous nitrogen flow of $0.21 / \mathrm{min}$ through a $1 \mathrm{~mm} \varnothing$ tube terminating $1 \mathrm{~cm}$ from both antennae. An electrically controlled valve allowed the replacement of this nitrogen flow by odorant pulses. In this way, mechanical stimulation was kept constant. Responses to five $300 \mathrm{msec}$ pulses at $30 \mathrm{sec}$ intervals to increasing concentrations of ethyl acetate (EA) diluted in paraffin oil (Fluka, Neu-Ulm, Germany) were recorded and analyzed on pClamp 6.0 software. Concentrations are expressed as $\mathrm{v} / \mathrm{v}$ dilution factor.

Behavioral tests. Screening for mosaics was done under $\mathrm{CO}_{2}$ anesthesia. For behavioral tests, fully recovered mosaics $(n=70)$ and sibling controls $(n=70)$ were tested individually in a T-maze apparatus (Tully and Quinn, 1985). Before the test, each fly was kept overnight in a humidified chamber without food. The fly was allowed $30 \mathrm{sec}$ to choose between a vial $(17 \times 100 \mathrm{~mm}$; model 2051 , Becton Dickinson, Franklin Lakes, NJ ) containing a filter paper $(4 \times 1 \mathrm{~cm})$ soaked in $100 \mu \mathrm{l}$ of ethyl acetate diluted in paraffin oil (odorant vial) and another containing paraffin oil (control vial). Flies were prevented to contact the filter paper by a cotton barrier. Chemicals were of the maximum available purity from Fluka (Buchs, Switzerland). Vials were used only once, and their relative position in the T-maze was at random. Each fly was tested under the whole range of odorant concentrations allowing $5 \mathrm{~min}$ between exposures. Experimental and control flies alternated in each test series. Control flies in these experiments are sibling animals that do not exhibit a mutant clone. Experiments were performed in the dark to avoid visual interference, and the apparatus was kept horizontal to avoid influences from the geotaxic response. Cases of no choice, when the fly remained in the central chamber, were discarded. The response index is calculated as the number of positive responses (flies in the odorant tube) minus the number of negative responses (flies in the paraffin oil tube) divided by the total. Values of this index range from 1 (full attraction) to -1 (full repulsion), while 0 is considered indifference or equilibrium between attraction and repulsion (Ayyub et al., 1990). All tests were performed in a room at $25^{\circ} \mathrm{C}$ and $80 \%$ humidity to ensure constant vapor pressure.

Statistical analysis. In the morphological studies, values from gig and contralateral sides of the same mosaic have been compared using paired statistical tests. Nonpaired tests were used when comparing gig mosaics and their sibling controls. The particular test used is indicated in the corresponding tables and text. Data are presented as mean \pm SEM. In the behavioral studies, the olfactory index was determined in single individual tests until a total of 70 cases per concentration and genotype were reached. Olfactory index values were submitted to the $\chi^{2}$ test to identify peak values significantly different from indifference $(0.0$ olfactory index value). The comparison between mutant and control olfactory index values per concentration was subject to the Mann-Whitney $U$ test. All statistical analyses were done with the SPSS 8.0 software. 

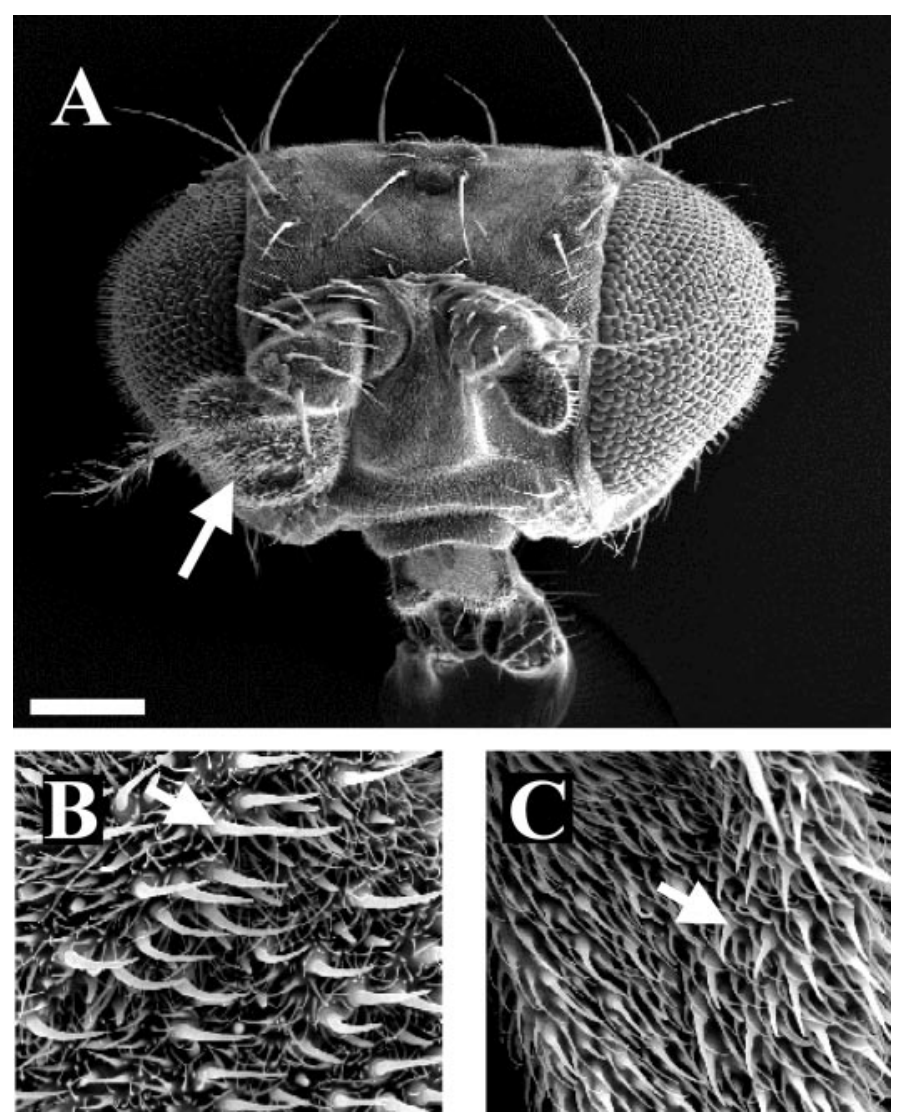

Figure 1. Morphology of antennal gig mosaics. Scanning electron microscope images from a $\mathrm{M}^{+}$gig antennal clone. $A$, Frontal view of a mosaic head showing the size difference between the gig (arrow) and contralateral antennae. $B$, gig sensillae are also larger than those from the contralateral side $(C)$ (arrows indicate trichoid sensillae). Scale bar: $A, 100$ $\mu \mathrm{m} ; B, C, 10 \mu \mathrm{m}$.

\section{RESULTS}

The gig antennae are larger than normal without changes in cell number

The structural and functional analyses were performed in antennal mosaics identified by the cuticular markers $m w h$ and $j v$, in addition to the gig phenotype, that served to determine the clone extent. Only mosaics affecting the whole antenna were used in this study. The mutant antenna is approximately twice the size of its contralateral counterpart (Fig. 1A). Despite this, the total number of sensillae in the mutant $(415 \pm 4)$ and contralateral $(374 \pm 15)$ antennae are not significantly different $(p>0.05 ; n=$ 3; Student's $t$ test); neither do they differ from those of wild-type C-S $(394 \pm 6)(p>0.05 ; n=6$; Mann-Whitney $U$ test $)$. In addition, the regional distribution of the different types of sensillae on the funiculus remains unaltered. Each sensillum, however, is enlarged in the mutant side in approximately the same proportion as the whole antenna (Fig. $1 B, C$ ). The effects of the mutation on the axons that travel along the antennal nerve (AN) and the brain centers were analyzed in paraffin- or resin-embedded material (Figs. 2, 3). These procedures cause different degrees of shrinkage. The AN, however, appeared always of a larger diameter than its contralateral homolog in the entire path toward the brain (Fig. 2A). AN axons were counted in both nerves of five mosaics using ultrathin sections taken midway along the projection (Fig. $2 B, C$ ). The resulting numbers indicate no significant
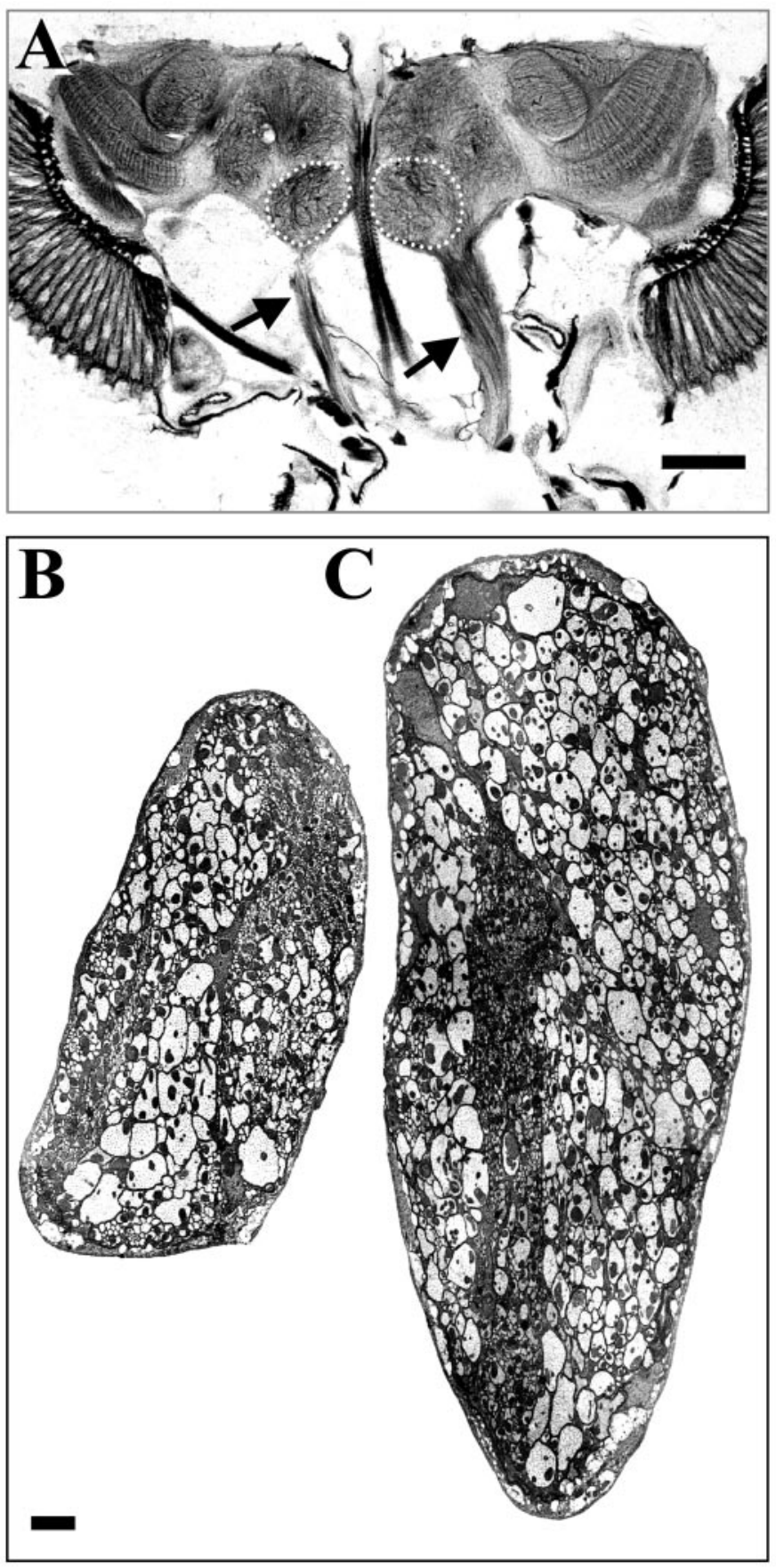

Figure 2. Antennal nerves of gig mosaics. A, Horizontal section from a gig mosaic head stained by Holmes-Blest silver impregnation. Note the diameter difference between normal (left) and gigas (right) nerves (arrows). See also Figure $3 B$. Anterior is down. $B, C$, Ultrathin frontal sections from normal $(B)$ and mutant $(C)$ antennal nerves from the same mosaic. The plane of section is approximately at the level of arrows in $A$. Note the caliber of gig neurons, approximately twice that of its contralateral homologs. Both nerves are flattened from the lateral sides, but the number of axons is not significantly different (Table 1). Dorsal is up in $B$ and $C$. Scale bars: $A, 50 \mu \mathrm{m} ; B, C, 2 \mu \mathrm{m}$.

difference between mutant and normal sides of each mosaic (Table 1). The intra-individual variability in the number of axons, in either mosaics or wild type, is $5-10 \%$, the same range as interindividuals. Because the number of profiles is virtually the same in both nerves, it is evident that the much larger cross- 


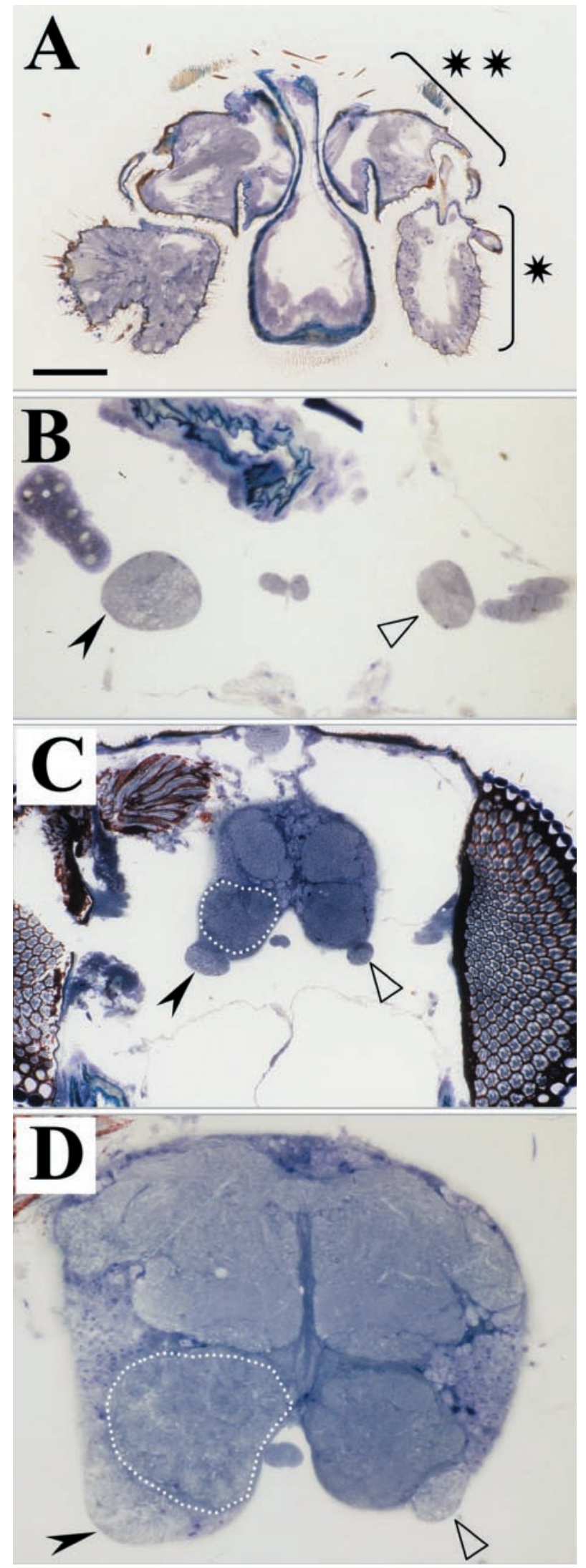

Figure 3. Brain structural effects of an antennal mosaic. Toluidinestained semithin $(1 \mu \mathrm{m})$ frontal sections through a single mosaic. $A$, Section at the level of antennal segments showing the funiculus (asterisk) and the pedicel (double asterisk). The mutant clone includes the whole antenna on the left side. $B$, Section at $3 \mu \mathrm{m}$ from that shown in $A$, showing
Table 1. Number of axons per antennal nerve

\begin{tabular}{|c|c|c|c|c|c|c|}
\hline Antenna & $\begin{array}{l}\text { Clone } \\
1\end{array}$ & $\begin{array}{l}\text { Clone } \\
2\end{array}$ & $\begin{array}{l}\text { Clone } \\
3\end{array}$ & $\begin{array}{l}\text { Clone } \\
4\end{array}$ & $\begin{array}{l}\text { Clone } \\
5\end{array}$ & Mean \pm SEM \\
\hline gig & 1152 & 1403 & 1654 & 1512 & 1815 & $1473 \pm 113$ \\
\hline Contralateral & 1349 & 1219 & 1403 & 1316 & 1572 & $1372 \pm 58$ \\
\hline
\end{tabular}

Mosaics were obtained in the genotype $m w h$ jv gig ${ }^{109} \mathrm{red} \mathrm{ro} / \mathrm{M}(3)^{i 55}$. Cases 2, 3, and 4 were male. The mutant side was left in mosaics 1,2 and 4 . Sections used for axon counts were as shown in Figure 2. These values are not significantly different between gig and contralateral nerves (Student's $t$ test).

sectional area of the mutant $\mathrm{AN}$ is attributable to the increased axon caliber. An obvious exception must be the motoneurons innervating the antennal muscles because they have a separate lineage from the olfactory cells and, consequently, are not included in the mutant clone. Their number, however, is very small compared with the sensory axons, as deduced from Wallerian degeneration experiments (Stocker, 1979).

The effects of mutant antennal clones in brain centers were examined in serial semithin sections. The antennal lobe (AL), the primary target of AN projection, is noticeably enlarged in the brains of all mosaics, whereas other brain nuclei are not overtly modified at this level of observation (Fig. 3). The AL ipsilateral to the mutant antenna shows a larger size increment than its contralateral equivalent. To analyze this structural effect in greater detail, we used HRP, Golgi, and GFP tracing methods.

\section{The gig sensory afferents project to their normal glomerular targets}

Many of the 43 AL glomeruli receive afferents from both antennae, one of the exceptions being the $\mathrm{V}$ glomerulus that is innervated by the ipsilateral appendage only. Within each glomerulus, synaptic contacts are established between axon terminals of sensory neurons and dendrites of AL neurons (Stocker et al., 1990; Anton and Homberg, 1999). In the wild type, sensory neurons exhibit a greater branching in the ipsilateral glomeruli than in the contralateral targets (Fig. 4A) (Stocker et al., 1990). This feature is also maintained by gig afferents, as shown by Golgi impregnations (Fig. 4B). In addition, the projection of gig afferents was monitored with the $n$-synaptobrevin-GFP reporter UNG6, driven by Gal4-72OK (Fig. 4C). These individuals express the reporter in a subset of sensory neurons that project to VM1, VM4, and DL1 glomeruli. In the eight mosaics studied, we found no differences between the projection patterns of gig and sibling controls. The only noticeable change was an increase in volume in the three glomeruli ipsilateral to the mutant antenna with respect to the contralateral homologs (Fig. 4C). The GFP-marked material corresponds to unfixed whole mounts in which no shrinkage is expected and, therefore, the measurements should be more precise than in fixed material. The ratio of volume increment is 1.3-1.6 depending on the glomerulus. It should be noted, how-

the antennal nerves in the mutant (solid arrowhead) and normal (open arrowhead) sides. $C$, Section at $5 \mu \mathrm{m}$ from $A$, showing the antennal nerves and lobes (stippled in the mutant side). $D$, Section at $9 \mu \mathrm{m}$ from $A$. Note the larger area of the antennal nerve and lobe in the mutant side. Both lobes are enlarged with respect to the wild type because of the bilateral projection of a fraction of sensory afferents. The increment, however, is larger in the lobe ipsilateral to the mutant antenna because not all afferents have bilateral projections, and branching is usually more abundant in the ipsilateral glomeruli. This differential branching is present in wild type and maintained in the mutant (Fig. 4). Scale bar: $A, C, 50 \mu \mathrm{m}$; $B, D, 25 \mu \mathrm{m}$. 

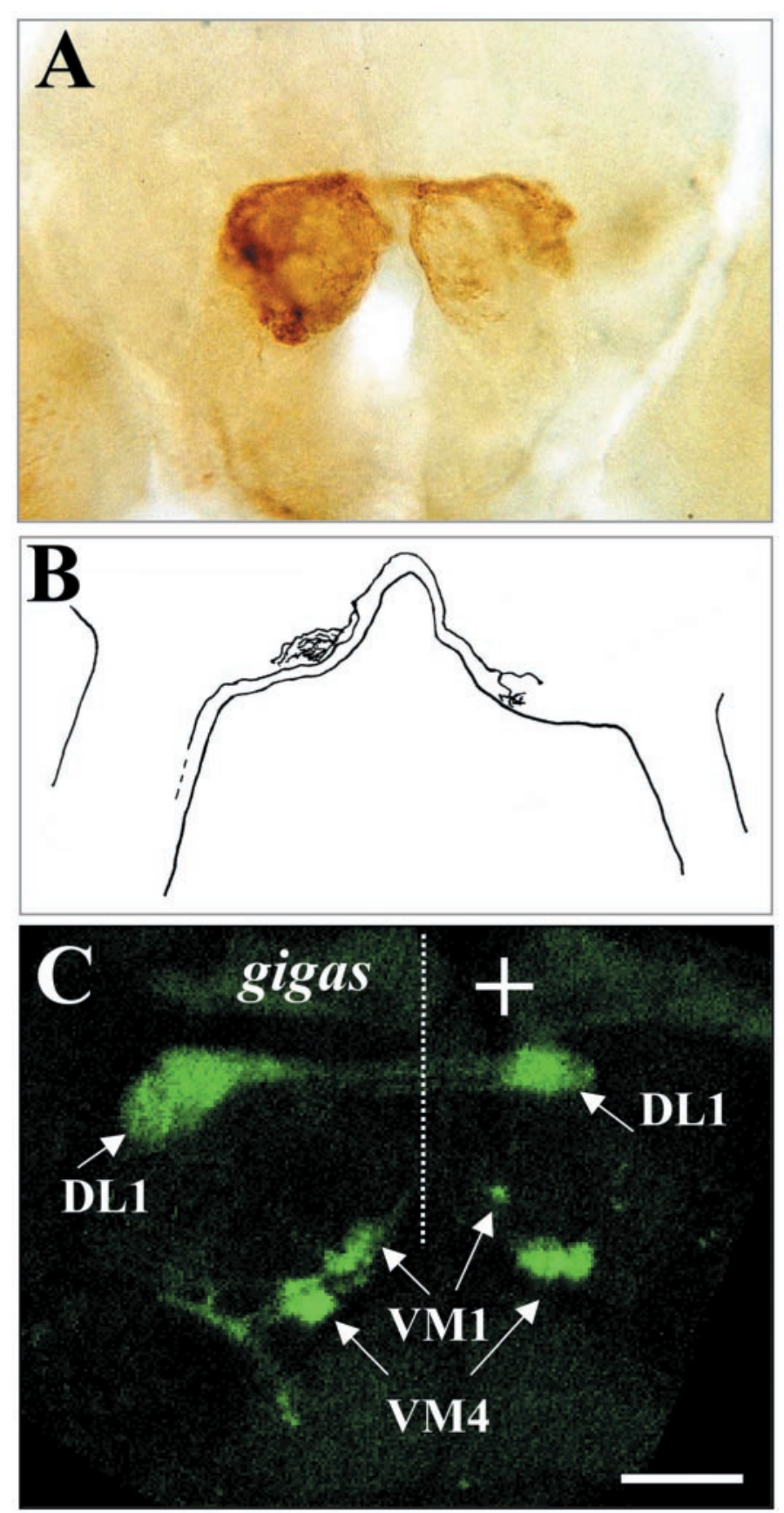

Figure 4. Projection pattern into antennal lobes. $A$, Frontal view of a whole mount C-S wild-type brain backfilled with HRP from the antenna on the left side. Note that most sensory afferents project and branch more intensively into the ipsilateral lobe. The $\mathrm{V}$ glomerulus is located in the ventral (down) apex of the lobe. Note that the contralateral locus is not marked by HRP. B, Camera lucida drawing of a gigas sensory neuron impregnated by the Golgi method. The mutant cell branches more profusely in the glomerulus ipsilateral to the mutant antenna (left side) as do normal cells. The amount of branching, however, is higher than in the wild type (Stocker et al., 1990). C, Frontal view of a confocal microscope section from the brain of a mosaic induced in a Gal4-72OK/UNG6 background. This Gal4 drives the expression of an $n$-Syn-GFP chimera in olfactory neurons that project to DL1, VM1, and VM4 glomeruli (arrows). The mutant afferents maintain this projection pattern. Note, however, the increased size of glomeruli ipsilateral to the mutant antenna. Lobes ipsilateral to mutant (gigas) and normal $(+)$ antennae are separated by a dotted line. Scale bar: $A, 65 \mu \mathrm{m} ; B, 40 \mu \mathrm{m} ; C, 30 \mu \mathrm{m}$.
Table 2. Volume increase of $\mathrm{V}$ glomeruli in gig mosaics

\begin{tabular}{lllll} 
Mosaic & gig & Contralateral & Ratio & Mean \pm SEM \\
\hline 1 & 15177 & 11674 & 1.30 & \\
2 & 11064 & 7002 & 1.58 & \\
3 & 17673 & 12806 & 1.38 & $1.42 \pm 0.07^{*}$
\end{tabular}

Measurements (in cubic micrometers) were obtained using the UAS- $n$ synaptobrevin-GFP signal driven by Gal4-C155 [genotype: Gal4-C155; UNG6/+; $m w h$ jv gig ${ }^{109}$ red ro/M(3) ${ }^{i 55}$ ] under confocal microscope images. Mosaic 2 was male. *Statistically significant difference at $p<0.01$; Student's $t$ test.

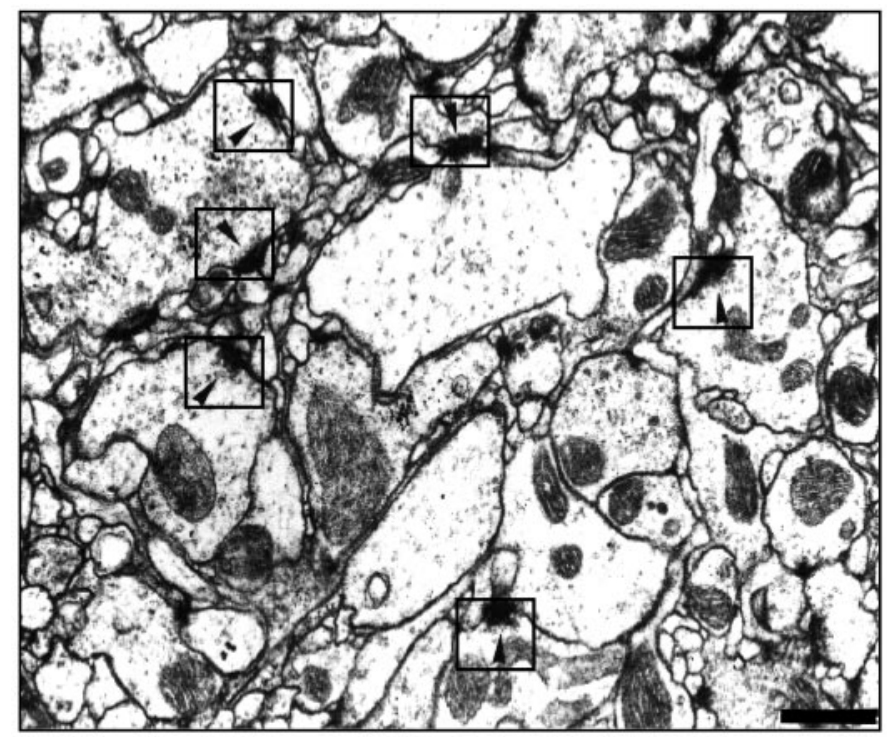

Figure 5. Synaptic contacts in the olfactory neuropil. Electron micrograph of a V glomerulus innervated from a gig antenna. Several presynaptic active zones are marked by framed arrowheads. Their size is normal, but their number is increased (Table 2). Scale bar, $500 \mathrm{~nm}$.

ever, that because many olfactory sensory neurons have bilateral projections, and the extent of branching is different for each type of neuron, the net effect of the mutation might be underestimated. To clarify this, we analyzed the $\mathrm{V}$ glomerulus in detail because it receives ipsilateral projections only.

\section{Synapse number is increased in a glomerulus that receives gig afferents}

We measured the $\mathrm{V}$ glomerulus volume on a series of confocal optical sections from mosaics expressing $n$-Syb/GFP driven by Gal4-C155. A volume ratio between both V glomeruli was calculated for each mosaic. The data show a significant increment of $42 \%$ (Table 2). A larger glomerular volume may not be functionally relevant, however, if the density of synapses is downregulated. We therefore performed a quantitative analysis of synapses using the disector technique (see Materials and Methods) in serial ultrathin sections of this glomerulus (Fig. 5). The separation between disectors $(140 \mathrm{~nm})$ was adjusted to the average size of synaptic specializations in this region. The number of disectors used, 25-30 in each glomerulus in every mosaic, represents $\sim 5 \%$ of the total $\mathrm{V}$ volume. The data from four mosaics reveal that the average density of synapses, $\sigma$, is $\sim 60 \%$ greater in mutant ipsilateral versus contralateral V glomeruli (Table 3). Combining these two sets of data, we estimate the increment in synapse number $(\Delta N)$ attributable to the arrival of mutant afferents in this particular glomerulus as $\Delta N=\Delta \sigma \times \Delta V(1.64 \times 1.42=2.33)$. 


\begin{tabular}{|c|c|c|c|c|c|c|c|c|}
\hline \multirow[b]{2}{*}{ Mosaic } & \multicolumn{3}{|l|}{ gig } & \multicolumn{3}{|c|}{ Contralateral } & \multirow{2}{*}{$\begin{array}{l}\text { Ratio gig/ } \\
\text { contral. }\end{array}$} & \multirow{2}{*}{$\begin{array}{l}\text { Mean ratio } \\
\pm \text { SEM }\end{array}$} \\
\hline & $n$ & $\mathrm{~V}$ & $\sigma$ & $n$ & $\mathrm{~V}$ & $\sigma$ & & \\
\hline 1 & 258 & 52.8 & 4.88 & 200 & 68.7 & 2.91 & 1.67 & \\
\hline 2 & 39 & 20.0 & 1.95 & 20 & 17.3 & 1.15 & 1.70 & \\
\hline 3 & 46 & 43.3 & 1.06 & 27 & 39.7 & 0.68 & 1.56 & \\
\hline 4 & 178 & 56.3 & 3.16 & 122 & 62.5 & 1.95 & 1.62 & $1.64 \pm 0.03^{*}$ \\
\hline \multicolumn{9}{|c|}{$\begin{array}{l}\text { The total number of synapses }(n) \text { identified in the sampled volume }(\mathrm{V} \text { in cubic } \\
\text { micrometers) of each } \mathrm{V} \text { glomerulus is shown. A total of } 107 \text { and } 112 \text { dissectors were } \\
\text { analyzed in the normal and mutant glomeruli, respectively. Mosaics } 3 \text { and } 4 \text { were } \\
\text { males. }\end{array}$} \\
\hline
\end{tabular}

Table 4. Density of terminals with large-dense-core vesicles (number/ $\mu \mathbf{m}^{3}$ )

\begin{tabular}{lll} 
Mosaic & gigas & Contralateral \\
\hline 1 & 1.27 & 1.64 \\
2 & 0.79 & 1.19 \\
3 & 1.67 & 1.05 \\
4 & 1.18 & 1.06 \\
\hline Mean \pm SEM & $1.23 \pm 0.18$ & $1.23 \pm 0.14$
\end{tabular}

Mosaics and sections were the same as those used in Table 3. These values are not significantly different between gig and contralateral V glomeruli (Student's $t$ test).

The mutant ipsilateral $\mathrm{V}$ glomerulus, thus, exhibits twofold to threefold more synapses than the contralateral homolog. It should be noted that synapse estimations do not discriminate between those established by incoming mutant sensory neurons and those formed by neurons intrinsic to the glomerulus that are genetically normal. Assuming that genetically normal neurons will not modify their number of synapses substantially, as it is the case in optic lamina cartridges (Canal et al., 1994), the value of 2.33 may underestimate the actual increment in synapses produced by the gig sensory afferents (see Discussion).

The choice of the $\mathrm{V}$ glomerulus for the quantitative synaptic study avoids the problem of contralateral projections from normal sensory neurons. It does not prevent, however, the problem of discrimination between sensory and intrinsic synapses. To identify selectively the two types of synapses is not feasible at present. An alternative approach, however, is to analyze boutons that can be identified at the EM by their vesicle morphology, the largedense-core vesicles. These are probable homologs of serotoninergic-imnunoreactive terminals described in other insect species (Sun et al., 1993), and we found them in the ultrathin sections from the $\mathrm{V}$ glomerulus used for disector analysis. Table 4 shows that their density is not different in mutant versus contralateral V glomeruli. Their size appeared normal as well (data not shown). Taken together, the observations in the visual system and the peptidergic terminals of the $\mathrm{V}$ glomerulus support the assumption that AL interneurons do not increase their synapse number when innervated by gigas sensory neurons (see Discussion).

\section{The gig olfactory neurons exhibit normal sensory transduction}

To test the functional performance of mutant antennae, we recorded EAGs after stimulation with ethyl acetate $\left(10^{-5}\right.$ to $10^{-1} \mathrm{v} / \mathrm{v}$ ) (Fig. 6). Recordings were obtained simultaneously from mutant and normal antennae in mosaics and sibling controls.
A

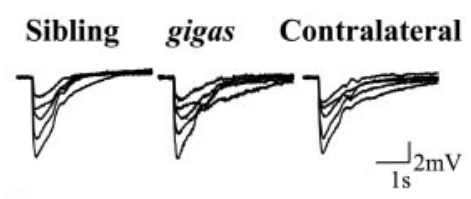

B

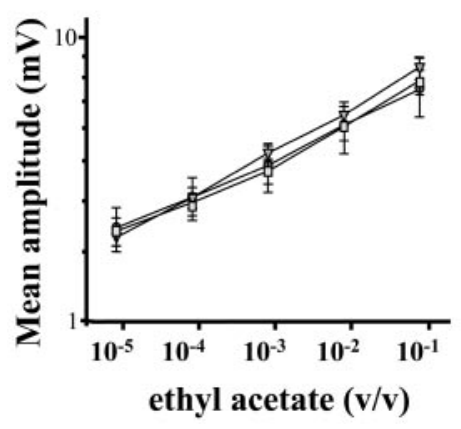

Figure 6. Olfactory transduction tests. $A$, EAGs obtained in response to five ethyl acetate concentrations $\left(10^{-5}\right.$ to $\left.10^{-1}\right)$ from sibling controls $(n=6)$, and gig mosaics $(n=5)$. Amplitudes correlate with stimulus concentrations. B, Logarithmic plot of EAG amplitudes from sibling controls (filled inverted triangles), contralateral (filled circles), and gig mosaics (open squares). No significant difference is observed between mutant and controls. Error bars indicate SEM.

The amplitude of the sensory receptor potential increased according to the stimulus concentration. In no case were differences in amplitude or kinetics detected in mutant versus control EAG. Additional EAGs were obtained after stimulation with benzaldehyde $\left(10^{-4}\right.$ to $\left.10^{-1} \mathrm{v} / \mathrm{v}\right)$, also with no detectable differences between gig and controls (data not shown). The lack of effects is consistent with the coordinated increment of all cellular constituents (e.g., odorant receptors and downstream elements) expected from the increased ploidy of gig cells. In this experimental design and for the odorants tested, therefore, gig does not produce detectable changes in olfactory transduction.

\section{Olfactory behavior is modified in gig mosaics}

To determine possible mutant effects on behavior, we performed olfactory tests with EA over a broad concentration range $\left(10^{-12}\right.$ to $\left.10^{-1} \mathrm{v} / \mathrm{v}\right)$. In this test, nonconditioned single flies in a T maze apparatus are allowed to choose between a given concentration of odorant and the solvent (see Materials and Methods). Previous experiments showed that results are gender- and mutant sideindependent, thus allowing data pooling. Response index values between 0.0 and 1 indicate attraction, those between 0.0 and -1 indicate repulsion, and 0.0 is considered as indifference (Ayyub et al., 1990). The specific response is stimulus concentration- and genotype-dependent. In addition, the design of the apparatus and the conditions of the animals may cause variations of index values (see below). Nevertheless, Drosophila adults exhibit the same response trend to virtually all odorants, indifference to low concentrations, attraction to moderate concentrations, and repulsion to high concentrations (Ayyub et al., 1990; Alcorta, 1991).

The choice of control animals is a key factor when behavioral differences of genetic origin are under study. In this report, control and experimental animals are siblings. Thus, genetic background as well as rearing conditions are as similar as possible between both groups. It should be noted, however, that control animals are not a standard wild type (e.g., C-S). In this context, 

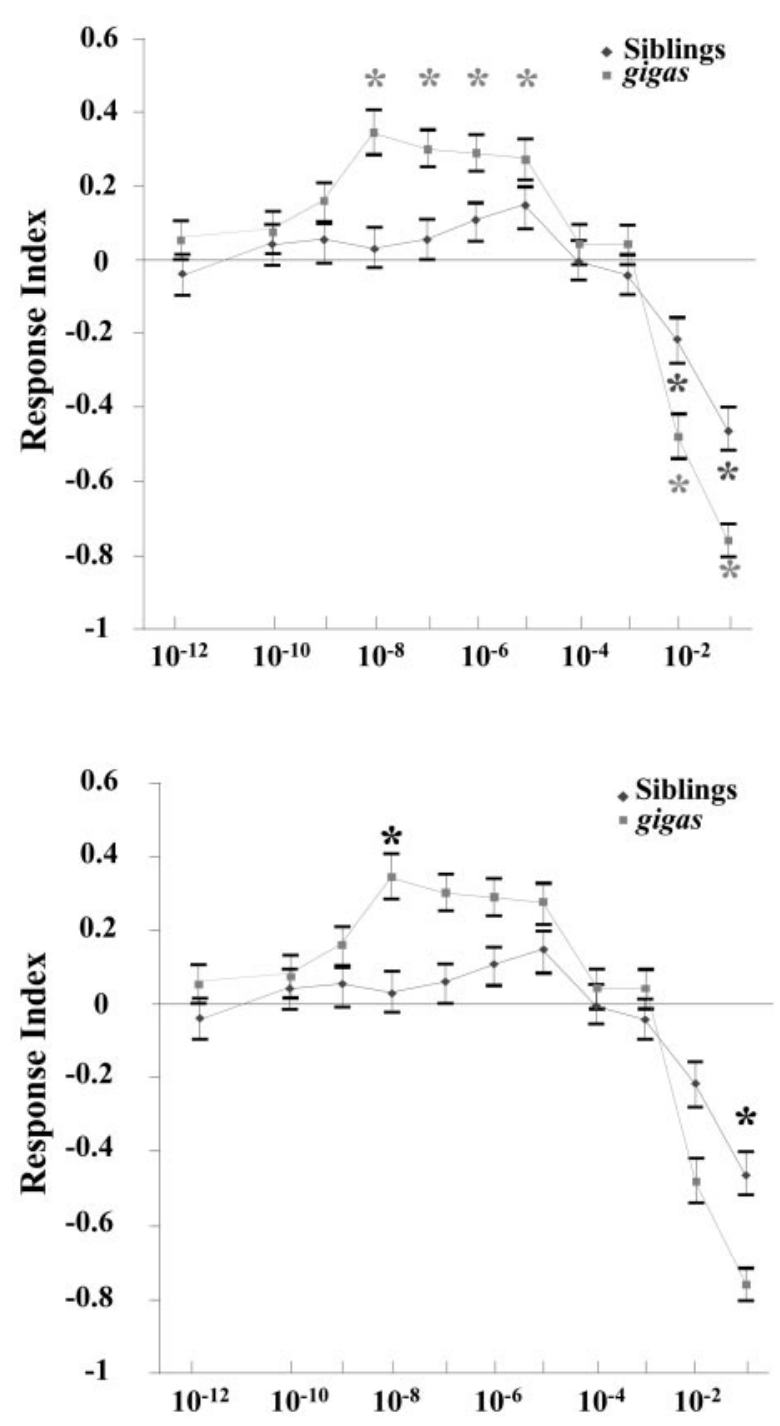

Figure 7. Olfactory behavioral responses. Dose-response curves from experimental mosaics (gray squares) and sibling controls (black diamonds). Values represent the mean response $( \pm$ SEM) from 70 individuals per odorant concentration. Olfactory index values can range from 1 (full attraction) to -1 (full repulsion), and 0 is considered the indifference line. $A$, Olfactory responses significantly different ( $\chi^{2}$ test) from indifference. These were found at $10^{-1}$ and $10^{-2}$ in sibling controls $(p<0.05)$ and mosaics $(p<0.01)$, and at $10^{-8}$ to $10^{-5}$ in the mosaics $(p<0.05, p<$ $0.01, p<0.05, p<0.05$, respectively). Note the significant attraction response of mosaics at concentrations that yield indifference in the controls $\left(10^{-8}\right.$ to $\left.10^{-5}\right)$. B, Significant differences (Mann-Whitney $U$ test) between mosaics and sibling controls at $10^{-8}$ and $10^{-1}$ concentrations $(p<0.05$ and $p<0.01$, respectively). Note the augmented responses in the mutant.

the profile of the olfactory response to EA in the sibling controls (Fig. 7), although it follows the same trend as all odorant responses, differs from some reports on C-S (Ayyub et al., 1990). The main difference is the lack of a statistically significant $\left(\chi^{2}\right.$ test) attractive response to any concentration. This difference could be attributable to the conditions of the test, the apparatus design, or the genotype. To discriminate among these possibilities, we tested $\mathrm{C}-\mathrm{S}$ adults in the same apparatus. The index value obtained for the exposure to $10^{-4} \mathrm{EA}$ was $0.56 \pm 0.01$, in line with the values obtained for the sibling controls. That is, a weak, not statistically significant, attractive response. In addition, failure to detect strong attraction to EA at $10^{-3}$ and $10^{-4}$ was reported also in an independent study (Alcorta, 1991). Thus, the previously reported strong attractive response of C-S to EA (Ayyub et al., 1990), seems to be detected only with the experimental design used in that report, and comparison with the sibling controls of the present report are not justified. Concerning the repulsive responses of the sibling controls, a significant peak ( $\chi^{2}$ test) is detected at $10^{-1}$.

With gig mosaics, the normal trend of responses is observed, albeit with two noticeable differences: the odorant concentrations that elicit them and their intensity (Fig. 7). The significant $\left(\chi^{2}\right.$ test) attraction in mosaics develops at concentrations that result in indifference in control individuals (see $10^{-8}$ to $10^{-5}$ range in mosaics vs controls) (Fig. 7A). The odorant concentration for the attractive response in mosaics is $10^{-8} \mathrm{v} / \mathrm{v}$, three orders of magnitude lower than that for sibling controls in which only a mild attractive, albeit statistically not significant, response is detected at $10^{-5}$.

In addition to this change in the olfactory profile, the intensity of the responses is higher in the mosaic flies (Mann-Whitney $U$ test; $p<0.05)$. The effect is detected at attractive, $10^{-8}$, as well as at repulsive, $10^{-1}$, concentrations at which the indexes are almost double in gig with respect to siblings (Fig. $7 B$ ). Overall, the profile of responses in the mosaics is consistent throughout the range of concentrations. These data illustrate one of the functional consequences of the morphological changes described above and show that gig olfactory receptor neurons cause a modification of perception that can be described as an increment in sensitivity. Complementary data on the synaptic effects associated to loss of olfactory sensitivity are described in an accompanying report (Devaud et al., 2001).

\section{DISCUSSION}

Here we show that an increase in synapse number endows Drosophila with a greater sensitivity of perception. The gene gigas has been instrumental to generate mosaics with a mutant antenna. In this type of individuals, the structural features of the main olfactory centers, the sensilla and the glomeruli, are analyzed. Finally, the behavioral effect of modifying sensory neurons is tested in the odorant preference test.

\section{Structural effects}

The number of olfactory sensillae and afferents are not modified by the gigas mutation. Although larger in size, the mutant neurons maintain their projection pattern to their appropriate glomeruli. These features are consistent with data in the visual system (Canal et al., 1994) but differ from those obtained in the mechanosensory system (Canal et al., 1998). Whereas olfactory and visual sensory neurons maintain their normal targets, mechanosensors project beyond their normal sites, reaching additional, more distant, mechanosensory centers. It is likely that the differential effect results from the structural limitation imposed by the glia. In the case of photoreceptors, their targets in the lamina are cartridges enveloped by glia, whereas mechanoreceptor centers are not. Similar to the lamina cartridges, the olfactory glomeruli of Drosophila are delimited by a glial envelope (our unpublished data). Although this glial cover is not as compact as in the moth Manduca sexta (Tolbert and Oland, 1990) or in the honeybee Apis mellifera (Gascuel and Masson, 1991), the results with gig suggest that glial constraints may prevent the invasion of adjacent glomeruli by mutant sensory afferents. Studies in vertebrates (González et al., 1993; Valverde, 1999) and invertebrates (Krull 
et al., 1994; Oland et al., 1998) illustrate the role of the ensheathing glia as a physical barrier, limiting branch growth to the inside of each glomerulus, and/or as a chemical barrier acting through extracellular matrix components. In addition, a variety of molecules (e.g., Fasciclin II-like, Semaphorins, Slit, Robo, NO) that are expressed by subsets of olfactory axons have been postulated to contribute to proper targeting (Tolbert, 1998; De Castro et al., 1999; Li et al., 1999; Pasterkamp et al., 1999). Any of these possibilities, glial cell restrictions, specific receptor expression and guidance cues, or their combination, may account for the restriction of mutant axons to their normal targets. These mechanisms appear to be normal in gig olfactory projections.

The most important effect of gigas in the context of neural structure is the increment in synapse number, $\Delta \mathrm{N}$. In the olfactory system, the increase ratio (2.3) appears to be somewhat lower than in the visual system (2.7) (Canal et al., 1994). It is likely that the difference reflects an underestimation for the olfactory system, because it is impossible to discriminate between afferent and intrinsic synapses. In the visual system, discrimination throughout the serial sections was possible because neurons have stereotyped positions within each lamina cartridge. Nonetheless, a putative effect of the mutant input on the branching or synapse number of intrinsic AL neurons cannot be formally excluded in the experiments reported here. The only type of nonsensory terminals that we observed in the AL were those containing large-dense-core vesicles (see below). In the $\mathrm{V}$ glomerulus, at least, their number and size is not affected by the mutant afferents.

The maximum length of a synaptic specialization profile is $\sim 200 \mathrm{~nm}$, if both arms of the T-shaped structure are added. It should be noted that this value is fairly constant in olfactory and visual sensory neurons and is not altered by the mutant condition. The size is similar in the mouse cortex $(250 \mathrm{~nm})$ and relatively invariant among individuals (De Felipe et al., 1997; 1999). This constancy suggests that synapse size is determined, in vertebrates as well as invertebrates, by intrinsic properties of its constituents and possibly reflects a fundamental constraint to vesicle docking and exocytosis. Glomerular volume and density of synapses can vary greatly between individuals (Tables 2,3 ). This is a common finding in quantitative synaptic studies in all species that, probably, sustains the behavioral differences among individuals. In addition, the density of synapses appears to be heterogeneous within each olfactory glomerulus, as documented in the bee (Gascuel and Masson, 1991) and the cockroach (Malun, 1991). Although the EM sections obtained here were done on brains carefully oriented to ensure equivalence between the left and the right AL, the relative depth of section cannot be reproduced, in practice, between individuals. Thus, the variability shown in Table 3 represents the addition of two components: interindividual variability and intraglomerular heterogeneity. In this context, the use of mosaics offers the key benefit of intraindividual comparisons, and the consistent feature observed in the four mosaics analyzed is a $>1$ ratio between gigas and contralateral sides.

\section{Functional effects of $\Delta \mathbf{N}$}

The sensation of the stimulus does not appear to be altered in gig. Receptor potentials indicate the population of receptor molecules activated by stimulant binding (Ayer and Carlson, 1992). In the mutant and control antennae, the amplitude of these potentials is odorant concentration-dependent. These observations are consistent with a proportional increment in the number of all molecule types, expected from an increased ploidy of the mutant cell. Receptor potential amplitudes are independent of cell size and are transmitted passively along the neuron membrane to the site of action potential generation. At this point, the gig neurons probably exhibit the first differences versus the contralateral homologs. Because axon caliber is larger in gig, it is expected that velocity of propagation will be faster than normal. Convergence of ipsilateral and contralateral inputs in each glomerulus thus, might register slight asynchrony, the functional effects of which in olfactory processing are unknown. In this context, it is plausible that if mosaics could be generated with both antenna being mutant, the behavioral changes would be more extreme. Producing this type of mosaics, however, is not yet feasible.

Odorant sensation activates specific combinations of glomeruli (Joerges et al., 1997; Rubin and Katz, 1999). Because the connectivity in gigas is not altered, it is reasonable to assume that the glomerular code will also remain unchanged, at least in terms of the combination of activated glomeruli. Changes, however, will be expected in the intensity of activation. It is thought that the larger the number of boutons on a neuron, the higher its capabilities for integration (Gulyas et al., 1999). In this context, neurons postsynaptic to mutant sensory cells are expected to modify their activation properties. Although glomerular activation maps cannot yet be visualized in Drosophila, odorants are perceived because they produce behavioral responses. The main difference, however, is the concentration at which each response is elicited and its magnitude. Odorant concentrations that normally cause indifference (e.g., $10^{-8}$ ) are clearly attractive to the mutant mosaics. At peak attractive and repellent concentrations, the mean response values are increased in gig; the increment is notably largest $(100 \%)$ for the repellent response. This result is consistent with the general observation that aversive responses are more extreme than attractive ones in all organisms (Izquierdo, 1984). The maintenance of this difference, in addition to the consistency of the behavioral olfactory profile, indicates that the integration of odorant stimuli is not overtly abnormal in $g$ ig.

An important aspect of odorant perception is the modulatory effect sustained by feedback mechanisms from higher areas of the brain. In honeybees (Rehder et al., 1987), cockroaches (Salecker and Distler, 1990), and moths (Kent et al., 1987), centrifugal serotonin-immunoreactive neurons project to most, if not all, glomeruli in the AL. These neurons serve as a feedback pathway in olfactory processing, controlling the responsiveness of projection AL neurons (Salecker and Distler, 1990; Sun et al., 1993). These modulatory effects are likely to have a significant impact on odor-dependent behaviors (Kloppenburg et al., 1999). Although equivalent neurons have not yet been described in Drosophila, our EM images show terminals with dense core vesicles of the same diameter as those found in serotoninergic terminals of Periplaneta americana (Salecker and Distler, 1990) and Manduca sexta (Sun et al., 1993). In gig mosaics, however, these terminals are not significantly increased in number (Table 4), suggesting that this putative neuromodulatory mechanism does not compensate the increased synaptic input from gig afferents.

The behavioral response of gig mosaics to the odorant preference tests can be described as an increment in perception sensitivity. We use the term "perception" to underline the fact that the odorant stimulus has been processed and results in a coherent behavior. Current opinion in the field of psychophysics considers perception to be a constructive process in which the individual generates it, presumably in integrative brain centers. Perception 
is also thought to be limited to stimuli that the individual is prepared to perceive (Seligman, 1971). The type of manipulation applied here to an insect allows analysis of these issues in a novel way. At present, within the limits of the model used and modifying primary sensory neurons only, we can conclude that increasing the number of synapses endows the organism with a greater perception sensitivity, leaving the processing of the stimulus mostly intact. In an accompanying report (Devaud et al., 2001), we address the relationship between synapse number and olfactory behavior in a complementary way. Both experimental approaches lead to the same result, a positive correlation between synapse number and sensory perception.

\section{REFERENCES}

Alcorta E (1991) Characterization of the electroantennogram in Drosophila melanogaster and its use for identifying olfactory capture and transduction mutants. J Neurophysiol 65:702-714.

Anton S, Homberg U (1999) Antennal lobe structure. In: Insect olfaction (Hansson BS ed), pp 98-125. Berlin: Springer.

Ayer Jr RK, Carlson J (1992) Olfactory physiology in the Drosophila antenna and maxillary palp: acj6 distinguishes two classes of odorant pathways. J Neurobiol 23:965-982.

Ayyub C, Paranjape J, Rodrigues V, Siddiqi O (1990) Genetics of olfactory behavior in Drosophila melanogaster. J Neurogenet 6:243-262.

Bailey CH, Kandel ER (1993) Structural changes accompanying memory storage. Annu Rev Neurosci 55:397-426.

Bausenwein B, Dittrich AP, Fischbach KF (1992) The optic lobe of Drosophila melanogaster. II. Sorting of retinotopic pathways in the medulla. Cell Tissue Res 267:17-28.

Blest AD, Davie PS (1980) Reduced silver impregnations derived from the Holmes technique. In: Neuroanatomical techniques. Insect nervous system (Strausfeld NJ, Miller TA, eds), pp 97-118. New York: Springer.

Canal I, Fariñas I, Gho M, Ferrús A (1994) The presynaptic cell determines the number of synapses in the Drosophila optic ganglia. Eur J Neurosci 6:1423-1431.

Canal I, Acebes A, Ferrús A (1998) Single neuron mosaics of the Drosophila gigas mutant project beyond normal targets and modify behavior. J Neurosci 18:999-1008.

De Castro F, Hu L, Drabkin H, Sotelo C, Chedotal A (1999) Chemoatraction and chemorepulsion of olfactory bulb axons by different secreted semaphorins. J Neurosci 19:4428-4436.

De Felipe J, Marco P, Fairén A, Jones EG (1997) Inhibitory synaptogenesis in mouse somatosensory cortex. Cereb Cortex 7:619-634.

De Felipe J, Marco P, Busturia I, Merchán-Pérez A (1999) Estimation of the number of synapses in the cerebral cortex: methodological considerations. Cereb Cortex 9:722-732.

Devaud J-M, Acebes A, Ferrús A (2001) Odor exposure causes central adaptation and morphological changes in selected olfactory glomeruli in Drosophila. J Neurosci 21: 6274-6282.

Ferrús A, García-Bellido A (1976) Morphogenetic mutants detected in mitotic recombination clones. Nature 260:425-426.

Ferveur JF, Savarit F, O' Kane CJ, Sureau G, Greenspan RJ, Jallon JM (1997) Genetic feminization of pheromones and its behavioral consequences in Drosophila males. Science 276:1555-1558.

Friedrich RW, Korsching SI (1998) Chemotopic, combinatorial, and noncombinatorial odorant representation in the olfactory bulb revealed using a voltage-sensitive axon tracer. J Neurosci 18:9977-9988.

Galizia CG, McIlwrath SL, Menzel R (1999) A digital threedimensional atlas of the honeybee antennal lobe based on optical sections acquired by confocal microscopy. Cell Tissue Res 295:383-394.

Gao Q, Yuan B, Chess A (2000) Convergent projections of Drosophila olfactory neurons to specific glomeruli in the antennal lobe. Nat Neurosci 3:780-785.

Gascuel J, Masson C (1991) A quantitative ultrastructural study of the honeybee antennal lobe. Tissue Cell 23:341-355.

González ML, Malemud CJ, Silver J (1993) Role of astroglial extracellular matrix in the formation of rat olfactory bulb glomeruli. Exp Neurol 123:91-105.

Goodman CS, Shatz CJ (1993) Developmental mechanisms that generate precise patterns of neuronal connectivity. Cell [Suppl] 72:77-98.

Gulyas AI, Megias M, Emri Z, Freund TF (1999) Total number and ratio of excitatory and inhibitory synapses converging onto single interneurons of different types in the CA1 area of the rat hippocampus. J Neurosci 19:10082-10097.

Hebb DO (1949) The organization of behavior. New York: Wiley.

Heisenberg M, Heusipp M, Wanke C (1995) Structural plasticity in the Drosophila brain. J Neurosci 15:1951-1960.

Hildebrand JG, Shepherd GM (1997) Mechanisms of olfactory discrim- ination: converging evidence for common principles across phyla. Annu Rev Neurosci 20:595-631.

Innocenti GM, Fiore L, Caminiti R (1977) Exhuberant projection into the corpus callosum from the visual cortex of newborn cats. Neurosci Lett 4:237-242.

Ito N, Rubin GM (1999) gigas, a Drosophila homolog of tuberous sclerosis gene product-2, regulates the cell cycle. Cell 96:529-539.

Izquierdo I (1984) Endogenous state dependency: memory depends on the relation between the neurohumoral and hormonal states present after training and the time of testing. In: Neurobiology of learning and memory (Lynch G, McGaug JL, eds), pp 333-350. New York: Guilford.

Joerges J, Küttner A, Galizia CG, Menzel R (1997) Representation of odours and odour mixtures visualized in the honeybee brain. Nature 387:285-288.

Kent KS, Hoskins SG, Hildebrand JG (1987) A novel serotoninimmunoreactive neuron in the antennal lobe of the sphinx moth Manduca sexta persists throughout postembryonic life. J Neurobiol 18:451-465.

Kloppenburg P, Ferns D, Mercer AR (1999) Serotonin enhances central olfactory neuron responses to female sex pheromone in the male sphinx moth Manduca sexta. J Neurosci 19:8172-8181.

Krull CE, Morton DB, Faissner A, Schachner M, Tolbert LP (1994) Spatiotemporal pattern of expression of tenascin-like molecules in a developing insect olfactory system. J Neurobiol 25:515-534.

Laissue PP, Reiter C, Hiesinger PR, Halter S, Fischbach KF, Stocker RF (1999) Three-dimensional reconstruction of the antennal lobe in Drosophila melanogaster. J Comp Neurol 405:543-552.

Laurent G (1999) A systems perspective on early olfactory coding. Science 286:723-728.

Li HS, Chen JH, Wu W, Fagaly T, Zhou L, Yuan W, Dupuis S, Jiang ZH, Nash W, Gick C, Ornitz DM, Wu JY, Rao Y (1999) Vertebrate slit, a secreted ligand for the transmembrane protein roundabout, is a repellent for olfactory bulb axons. Cell 96:807-818.

Maguire EA, Gadian DG, Johnsrude IS, Good CD, Ashburner J, Frackowiak RS, Frith CD (2000) Navigation-related structural change in the hippocampi of taxi drivers. Proc Natl Acad Sci USA 97:4398-4403.

Malun D (1991) Inventory and distribution of synapses of identified uniglomerular projection neurons in the antennal lobe of Pleriplaneta americana. J Comp Neurol 305:348-360.

Moser MB (1999) Making more synapses: a way to store information? Cell Mol Life Sci 55:593-600.

Oland LA, Pott WM, Higgins MR, Tolbert LP (1998) Targeted ingrowth and glial relationships of olfactory receptor axons in the primary olfactory pathway of an insect. J Comp Neurol 398:119-138.

Pasterkamp RJ, Ruitenberg MJ, Verhaagen J (1999) Semaphorins and their receptors in olfactory axon guidance. Cell Mol Biol 45:763-769.

Perrimon N (1998) New advances in Drosophila provide opportunities to study gene functions. Proc Natl Acad Sci USA 95:9716-9717.

Phelps CB, Brand AH (1998) Ectopic gene expression in Drosophila using GAL4 system. Methods: a companion to methods in enzimology $14: 367-379$

Rajan I, Cline HT (1998) Glutamate receptor activity is required for normal development of tectal cell dendrites in vivo. J Neurosci 18:7836-7846.

Rehder V, Bicker G, Hammer M (1987) Serotonin-inmunoreactive neurons in the antennal lobes and suboesophageal ganglion of the honeybee. Cell Tissue Res 247:59-66.

Rodrigues V, Pinto L (1989) The antennal glomerulus as a functional unit of odor coding in Drosophila melanogaster. In: Neurobiology of sensory systems. (Singh RN, Strausfeld NJ, eds), pp 387-396. New York: Plenum.

Rubin BD, Katz LC (1999) Optical imaging of odorant representations in the mammalian olfactory bulb. Neuron 23:499-511.

Salecker I, Distler P (1990) Serotonin-immunoreactive neurons in the antennal lobes of the American cockroach Periplaneta americana: light-and electron-microscopic observations. Histochemistry 94:463-473.

Shanbhag SR, Singh K, Singh RN (1995) Fine structure and primary sensory projections of sensilla located in the sacculus of the antenna of Drosophila melanogaster. Cell Tissue Res 282:237-249.

Seligman MEP (1971) Phobias and preparedness. Behav Ther 2:307-320.

Sterio DC (1984) The unbiased estimation of number and sizes of arbitrary particles using the disector. J Microsc 134:127-136.

Stimson DT, Ramaswami M (1999) Vesicle recycling at the Drosophila neuromuscular junction. Int Rev Neurobiol 43:163-189.

Stocker RF (1979) Fine structural comparison of the antennal nerve in the homeotic mutant antennapedia with the wild-type antennal and second leg nerves of Drosophila melanogaster. J Morphol 160: 209-222. 
Stocker RF (1994) The organization of the chemosensory system in Drosophila melanogaster: a review. Cell Tissue Res 275:3-26.

Stocker RF, Lienhard MC, Borst A, Fischbach KF (1990) Neuronal architecture of the antennal lobe in Drosophila melanogaster. Cell Tissue Res 232:237-248.

Sun XJ, Tolbert LP, Hildebrand JG (1993) Ramification pattern and ultrastructural characteristics of the serotonin-immunoreactive neuron in the antennal lobe of the moth Manduca sexta: a laser scanning confocal and electron microscopic study. J Comp Neurol 338:5-16.

Sweeney ST, Broadie K, Keane J, Niemann H, O’Kane CJ (1995) Targeted expression of tetanus toxin light chain in Drosophila specifically eliminates synaptic transmission and causes behavioral defects. Neuron 14:341-351.

Tolbert LP (1998) Olfactory development in invertebrates. On the scent of central developmental issues. Ann NY Acad Sci 30:95-103.
Tolbert LP, Oland LA (1990) Glial cells form boundaries for developing insect olfactory glomeruli. Exp Neurol 109:19-28.

Tully T, Quinn W (1985) Classical conditioning and retention in normal and mutant Drosophila melanogaster. J Comp Physiol [A[] 157:263-277.

Valverde F (1967) Apical dendritic spines of the visual cortex and light deprivation in the mouse. Exp Brain Res 3:337-352.

Valverde F (1999) Building an olfactory glomerulus. J Comp Neurol 415:419-422.

Vaux DL, Korsmeyer SJ (1999) Cell death in development. Cell 96:245-254.

Vosshall LB, Wong AM, Axel R (2000) An olfactory sensory map in the fly brain. Cell 102:147-159.

West MJ (1999) Stereological methods for estimating the total number of neurons and synapses: issues of precision and bias. Trends Neurosci 22:51-61. 